\title{
THE EISENBUD-EVANS \\ GENERALIZED PRINCIPAL IDEAL THEOREM AND DETERMINANTAL IDEALS
}

\author{
WINFRIED BRUNS
}

\begin{abstract}
In [2] Eisenbud and Evans gave an important generalization of Krull's Principal Ideal Theorem. However, their proof, using maximal Cohen-Macaulay modules, may have limited the validity of their theorem to a proper subclass of all local rings. (Hochster proved the existence of maximal Cohen-Macaulay modules for local rings which contain a field, cf. [4]). In the first section we present a proof which is simpler and guarantees the Generalized Principal Ideal Theorem for all local rings. The main result of the second section was conjectured in [2]. Under a hypothesis typically being satisfied for the most important fitting invariant of a module, it improves the Eagon-Northcott bound [1] on the height of a determinantal ideal considerably. Finally we will discuss the implications of a recent theorem of Faltings [3] on determinantal ideals.
\end{abstract}

1. The Generalized Principal Ideal Theorem. We recall some notations from [2]. Let $R$ be a commutative noetherian ring, and $M$ a finitely generated $R$-module. The order ideal $M^{*}(x)$ of an element $x \in M$ is given by

$$
M^{*}(x):=\left\{f(x): f \in M^{*}\right\},
$$

where $M^{*}$ denotes the dual $\operatorname{Hom}_{R}(M, R)$ of $M$. Since $M$ is finitely presented, the formation of $M^{*}(x)$ commutes with flat ring extensions, in particular with localizations, completions, and the adjunction of indeterminates. The rank of $M$ is the maximum of $\operatorname{dim}_{R_{\mathfrak{p}} / \mathfrak{p} R_{\mathfrak{p}}} M_{\mathfrak{p}} / \mathfrak{p} M_{\mathfrak{p}}, \mathfrak{p}$ ranging over the minimal primes of $R$. For all unexplained notations and terminology we refer the reader to [7].

Theorem 1 below extends Theorem 1.1 of [2] to all (local) rings $R$. It was named "Generalized Principal Ideal Theorem" because one recovers Krull's Principal Ideal Theorem for elements $x_{1}, \ldots, x_{m} \in R$ from it by specializing $M$ to $R^{m}$ and $x$ to $\left(x_{1}, \ldots, x_{m}\right) \in R^{m}$. (Theorem 1 was called the "Eisenbud-Evans Principal Ideal Conjecture" in [5].)

THEOREM 1. Let $R$ be a noetherian ring, $M$ a finitely generated $R$-module, and $x \in M$. If there is a prime ideal $\mathfrak{p}$ of $R$ with $x \in \mathfrak{p} M_{\mathfrak{p}}$, then

$$
\text { ht } M^{*}(x) \leqslant \operatorname{rank} M \text {. }
$$

Proof. It is enough to prove ht $M_{\mathrm{q}}^{*}(x)<\operatorname{rank} M_{\mathrm{q}}$ for a prime ideal q of $R$ (with $x \in q R_{\mathrm{q}}$ ): By the way rank $M$ was defined, it cannot increase under localization, and ht $M^{*}(x)<$ ht $M_{\mathrm{q}}^{*}(x)$ simply because $\left(M^{*}(x)\right)_{\mathrm{q}}=M_{\mathrm{q}}^{*}(x)$.

Received by the editors July 23, 1980 and, in revised form, November 6, 1980.

1980 Mathematics Subject Classification. Primary 13 COS.

() 1981 American Mathematical Society 0002-9939/81/0000-0404/\$02.50 
Let us first assume that there is a prime ideal $q$ of $R$ such that $M_{\mathrm{q}}$ is a free $R_{\mathrm{q}}$-module and $x \in \mathrm{q} M_{\mathrm{q}}$. Then ht $M_{\mathrm{q}}^{*}(x) \leqslant \operatorname{rank} M_{\mathrm{q}}$ by Krull's Principal Ideal Theorem since $M_{\mathrm{a}}^{*}$ is generated by rank $M_{\mathrm{q}}$ elements.

In the general case we may assume that $R$ is local with maximal ideal $\mathfrak{p}$. We may even suppose that $R$ is a complete local ring, height and rank being stable under completion. Finally we can factor out a minimal prime ideal $q$ of $\boldsymbol{R}$ for which ht $M^{*}(x)=h t\left(M^{*}(x)+q\right) / q$, cf. [2]. So we only need to prove the theorem for universally catenarian local domains.

There are elements $e_{1}, \ldots, e_{m} \in M$ such that $x=a_{1} e_{1}+\cdots+a_{m} e_{m}$ with $a_{i} \in \mathfrak{p}$. Let $S$ denote the localization of $R\left[T_{1}, \ldots, T_{m}\right]$ with respect to the maximal ideal generated by $\mathfrak{p}$ and the indeterminates $T_{1}, \ldots, T_{m}$. The ideal

$$
\mathfrak{r}:=S\left(a_{1}+T_{1}\right)+\cdots+S\left(a_{m}+T_{m}\right)
$$

is a prime ideal of $S$ with $\mathfrak{r} \cap R=\{0\}$. Thus

$$
(M \otimes S)_{\mathrm{r}}=M_{(0)} \otimes S_{\mathrm{r}}
$$

is a free $S_{\mathrm{r}}$-module $\left(M_{(0)}\right.$ denotes the localization of $M$ with respect to the zero-ideal of $R$ ). The element

$$
y:=\left(a_{1}+T_{1}\right) e_{1}+\cdots+\left(a_{m}+T_{m}\right) e_{m}
$$

is contained in $\mathrm{r}(M \otimes S)$. By what has been shown above, $\operatorname{ht}(M \otimes S)^{*}(y)<$ rank $M \otimes S=$ rank $M$.

$S$ is a catenarian ring. Consequently, there is a prime ideal q of $S$ containing $(M \otimes S)^{*}(y)$ as well as $T_{1}, \ldots, T_{m}$, such that ht $q<\operatorname{rank} M+m$. Then $q$ must also contain a minimal prime ideal $\tilde{q}$ of $(M \otimes S)^{*}(x)=M^{*}(x) S$. All minimal prime ideals of $M^{*}(x) S$ are extended from prime ideals of $R$. Therefore

$$
\tilde{\mathrm{q}} \subset \tilde{\mathrm{q}}+S T_{1} \subset \cdots \subset \tilde{\mathrm{q}}+S T_{1}+\cdots+S T_{m}
$$

is a strictly ascending chain of prime ideals, whence

$$
\text { ht } M^{*}(x)=\text { ht } M^{*}(x) S<\text { ht } \tilde{\mathrm{q}}<\operatorname{rank} M \text {. }
$$

The depth (or grade) of an ideal a with respect to an (arbitrary) $R$-module $N$, i.e., the length of a maximal $N$-sequence contained in $a$, is bounded above by ht $a$. Therefore Theorem 1 implies the corresponding inequality for depth. Being immediate consequences of Theorem 2.1 of [2], Corollaries 1.2 and 1.3 of [2] become valid for all local rings.

2. Determinantal ideals. As above, let $R$ be a commutative noetherian ring. The ideal generated by the determinants of the $t \times t$ submatrices of an $m \times n$ matrix $\varphi$ over $R$ is denoted by $I_{t}(\varphi)$ (with the usual conventions, $I_{t}(\varphi)=R$ for $t<0$ and $I_{t}(\varphi)=0$ for $\left.t>\min (m, n)\right)$. We define the kth fitting invariant $\mathrm{F}_{k}(M)$ of a finitely generated $R$-module $M$ [6] to be the ideal $\mathrm{I}_{n-k}(\varphi)$ where $\varphi$ represents a homomorphism $R^{m} \rightarrow R^{n}$ such that $M=$ Coker $\varphi$. The fitting invariants determine the level sets of the (locally constant) function assigning to each prime ideal $\mathfrak{p}$ the minimal number of generators of the $R_{\mathfrak{p}}$-module $M_{\mathfrak{p}}: \mathfrak{p} \supset \mathrm{F}_{k}(M)$ whenever $M_{\mathfrak{p}}$ cannot be spanned by fewer than $k+1$ elements. 
Let us say that $M$ has f-rank $r$ if $M_{\mathfrak{p}}$ is a free $R_{\mathfrak{p}}$-module of constant rank $r$ for all associated primes $\mathfrak{p}$ of $R$. The f-rank of $M$ is denoted by frk $M$. (This is the definition of rank proposed in [8].) In general, not every module has an f-rank. However, when $R$ is an integral domain or $M$ has a finite free resolution, then frk $M$ is defined, and, in the latter case, given by the Euler characteristic of a finite free resolution. The reader will check that frk $M=r$ if and only if $F_{r}(M)$ contains a nonzero divisor and $\mathrm{F}_{r-1}(M)=0$. Furthermore, in case frk $M=r$, a localization $M_{\mathfrak{p}}$ is a free $R_{\mathfrak{p}}$-module if and only if $\mathfrak{p} \not \supset \mathrm{F}_{r}(M)$. This property renders $\mathrm{F}_{r}(M)$ the most important of all fitting invariants and explains a great deal of our interest in a bound on ht $I_{t}(\varphi)$ under the condition $I_{t+1}(\varphi)=0$.

The classical bound on the height of determinantal ideals was given by Eagon and Northcott in [1, Theorem 3]:

$$
\text { ht } \mathrm{I}_{t}(\varphi)<\mathrm{EN}(m, n, t):=(m-t+1)(n-t+1)
$$

for $t=1, \ldots, \min (m, n)$, regardless of any hypothesis on $\varphi$ (except, of course, $\left.I_{t}(\varphi) \neq R\right)$. The "generic" case, in which $\varphi$ is a matrix of indeterminates over the integers, demonstrates that the Eagon-Northcott bound is optimal in general. One then has ht $\mathrm{I}_{t}(\varphi)=\mathrm{EN}(m, n, t)$ and, hence, ht $\mathrm{I}_{t}(\varphi) / \mathrm{I}_{t+1}(\varphi)=m+n-2 t+1$. The last equation presumably led Eisenbud and Evans to conjecture the following theorem [2, Conjecture 2.6]:

THEOREM 2. Let $R$ be a commutative noetherian ring, and $\varphi$ an $m \times n$ matrix over $R$. If $\mathrm{I}_{t}(\varphi) \neq R$ and $\mathrm{I}_{t+1}(\varphi)=0$, then

$$
\text { ht } I_{t}(\varphi) \leqslant m+n-2 t+1 \text {. }
$$

Proof. We use induction on $t$, and may restrict ourselves to complete local integral domains $R$ and ideals $I_{t}(\varphi)$ primary to the maximal ideal $m$ of $R$. Let

$$
\varphi=\left[\begin{array}{ccc}
x_{11} & \ldots & x_{1 n} \\
\vdots & & \vdots \\
x_{m 1} & \ldots & x_{m n}
\end{array}\right] .
$$

If there is an $x_{i j} \notin \mathrm{m}$, then one reduces the assertion to the case $t-1$ by applying elementary row and column operations to $\varphi$. So we may assume that all $x_{i j} \in \mathfrak{m}$, and, by induction on $n$, that there is a prime ideal $\mathfrak{p} \neq \mathfrak{m}$ containing $I_{t}\left(\varphi^{\prime}\right)$, where

$$
\varphi^{\prime}=\left[\begin{array}{ccc}
x_{11} & \cdots & x_{1 n-1} \\
\vdots & & \vdots \\
x_{m 1} & \cdots & x_{m n-1}
\end{array}\right]
$$

We claim ht $\mathrm{I}_{t}\left(\varphi^{\prime}\right) \leqslant n-t$. Consider $\varphi$ as a map $R^{m} \rightarrow R^{n}$ and, correspondingly, $\varphi^{\prime}$ as a map $R^{m} \rightarrow R^{n-1}$. Let $M:=\operatorname{Coker} \varphi$ and $M^{\prime}:=\operatorname{Coker} \varphi^{\prime} . M^{\prime}$ is isomorphic to $M / R_{\bar{e}}, e_{1}, \ldots, e_{n}$ denoting the elements of the canonical basis of $R^{n}$. Since $I_{t}(\varphi) \oplus \mathfrak{p}, M_{\mathfrak{p}}$ needs exactly $n-t$ generators. So does $M_{\mathfrak{p}}^{\prime}$ because $\mathrm{I}_{t}\left(\varphi^{\prime}\right) \subset \mathfrak{p}$. Necessarily $\bar{e}_{n} \in \mathfrak{p} M_{\mathfrak{p}}$, and ht $M^{*}\left(\bar{e}_{n}\right)<\operatorname{rank} M=n-t$ by Theorem 1. Regarding 
the determinantal relations of the columns of $\varphi$ as elements of $R^{n_{*}}$ which vanish on Im $\varphi$ (the submodule of $R^{n}$ generated by the rows of $\varphi$ ) we conclude $I_{t}\left(\varphi^{\prime}\right) \subset$ $M^{*}\left(\bar{e}_{n}\right)$, and obtain the claim.

In complete local domains the equation ht $a+\operatorname{dim} R / a=\operatorname{dim} R$ holds for all ideals a. Consequently Theorem 2 is settled once we have shown that $\operatorname{dim} R / I_{t}\left(\varphi^{\prime}\right)$ $=h \mathrm{I}_{t}(\varphi) / \mathrm{I}_{t}\left(\varphi^{\prime}\right) \leqslant m-t+1$.

LEMMA. Let $R$ be a local ring, and $\varphi$ an $m \times n$ matrix over $R$, whose last column consists of elements in the maximal ideal $m$ of $R$. Let $\varphi^{\prime}$ be the matrix formed by the first $n-1$ columns of $\varphi$. If $\mathrm{I}_{t}\left(\varphi^{\prime}\right)=0$, then

$$
\text { ht } I_{t}(\varphi) \leqslant m-t+1 \text {. }
$$

The lemma just extends Theorem 2.1 of [2] to all local rings. The following hint will enable the reader to prove it. Consider the transpose of $\varphi$ and adjoin a column to it:

$$
\tilde{\varphi}:=\left[\begin{array}{cccc}
x_{11} & \ldots & x_{m 1} & 0 \\
\vdots & & & \vdots \\
x_{1 n-1} & \ldots & x_{m n-1} & 0 \\
x_{1 n} & \cdots & x_{m n} & -1
\end{array}\right] .
$$

Now $\tilde{\varphi}$ and $\varphi$ are related in the same way as $\varphi$ and $\varphi^{\prime}$ in the proof of Theorem 2, and $\bar{e}_{m+1}=x_{1 n} \bar{e}_{1}+\cdots+x_{m n} \bar{e}_{m} \in \mathfrak{m} M$, the notations corresponding to those above.

Corollary 1. Let $R$ be as in Theorem 2, $\varphi$ an $m \times n$ matrix over $R$, and $\psi a$ $u \times v$ submatrix of $\varphi$ such that all coefficients of $\varphi$ outside $\psi$ generate a proper ideal of $R$. If $I_{t}(\varphi) \neq R$, then

$$
\text { ht } \mathrm{I}_{t}(\varphi) / \mathrm{I}_{t+k}(\psi)<\mathrm{EN}(m, n, t)-\mathrm{EN}(u, v, t+k)
$$

for all $k=0, \ldots, \min (u, v)-t+1$.

Proof. After the by now usual reduction to the case of a complete local domain, one applies Theorem 2 inductively to obtain the assertion in the case $\varphi=\psi$. Then one uses the lemma to complete the proof by induction on $(m+n)-(u+v)$.

Corollary 1, essentially predicted in [2], generalizes the Eagon-Northcott bound, to which it specializes for $\varphi=\psi, t+k=\min (m, n)+1$. It does not say (in general): ht $\mathrm{I}_{t}(\varphi)>\mathrm{EN}(m, n, t)$ implies ht $\mathrm{I}_{t+k}(\psi)>\mathrm{EN}(u, v, t+k)$. The corresponding statement for $\operatorname{dim} R-\operatorname{dim} R / I_{t}(\varphi)$ and $\operatorname{dim} R-\operatorname{dim} R / I_{t+k}(\psi)$, however, is always true (cf. [2, proof of Corollary 2.4]). Again the reader should observe that the inequalities for height imply the corresponding inequalities for depth.

We now return to the interpretation of determinantal ideals as fitting invariants. For a closed subset $A$ of $\operatorname{Spec} R$ we put

$$
\operatorname{codim} A:=\min \{\text { ht } \mathfrak{p}: \mathfrak{p} \in A\} .
$$

For every finitely generated $R$-module $M$

$$
\text { Nf } M:=\left\{\mathfrak{p} \in \operatorname{Spec} R: M_{\mathfrak{p}} \text { is not a free } R_{\mathfrak{p}} \text {-module }\right\}
$$


is a closed subset of Spec $R$ and consists of the prime ideals $\mathfrak{p} \supset F_{r}(M)$ in case frk $M=r$, as was noted above.

COROLlary 2. Let $R$ be as in Theorem 2, and $M$ a finitely generated $R$-module with an f-rank. Let $N$ be a second syzygy of $M$. If $M$ is not free, then

$$
\text { codim Nf } M<\text { frk } M+\text { frk } N+1 \text {. }
$$

Proof. Consider an exact sequence

$$
0 \rightarrow N \rightarrow R^{m} \stackrel{\varphi}{\rightarrow} R^{n} \rightarrow M \rightarrow 0
$$

and put $t:=n-$ frk $M$. Then $I_{t}(\varphi) \neq R, I_{t+1}(\varphi)=0$, frk $N=m-t$, and the conclusion follows from Theorem 2.

It would be extremely interesting to construct modules over regular local rings for which the bound in Corollary 2 is attained. It easy to write down examples with rank $N=0$ (equivalently, proj $\operatorname{dim} M=1$ ), and rather nontrivial ones with rank $N$ $=1$ can be found in [9], but we know of no such modules with rank $M>1$ and rank $N>1$.

In our last corollary $\mu(N)$ shall denote the minimal number of generators of an $R$-module $N$.

COROllaRY 3. Let $R$ be as in Theorem 2, $M$ a torsion-free $R$-module with an f-rank. Then

$$
\operatorname{codim} \text { Nf } M<\mu(M)+\mu\left(M^{*}\right)-2(\text { frk } M)+1 \text {. }
$$

Proof. Let $m:=\mu(M), n:=\mu\left(M^{*}\right)$, and choose generators $x_{1}, \ldots, x_{m}$ of $M$ and $f_{1}, \ldots, f_{n}$ of $M^{*}$. Let $\varphi$ be the $m \times n$ matrix $\left(f_{j}\left(x_{i}\right)\right)$. Then $\operatorname{Im} \varphi=M / U$, where $U$ is the kernel of the natural homomorphism $M \rightarrow M^{* *}$. Since $M$ has an f-rank, $U$ is a torsion module and thus $U=0$. It is easy to check that Nf $M=$ Nf Coker $\varphi$, frk Coker $\varphi=\mu\left(M^{*}\right)-$ frk $M$, and frk $\operatorname{ker} \varphi=\mu(M)-$ frk $M$. Now the conclusion follows at once from Corollary 2.

Theorem 3, which is a consequence of a theorem of Faltings [3], gives a better bound on ht $I_{t}(\varphi)$, provided $R$ is regular and $t$ is small compared to $m$ or $n$.

THEOREM 3. Let $R$ be a regular local ring, and $\varphi$ an $m \times n$ matrix over $R$. If $I_{t}(\varphi) \neq R$ and $\mathrm{I}_{t+1}(\varphi)=0$, then

$$
\text { ht } \mathrm{I}_{t}(\varphi)<\max (n, m-t+1) \text {. }
$$

Proof. Localizing with respect to a minimal prime ideal of $I_{t}(\varphi)$, we may suppose $I_{t}(\varphi)$ primary to the maximal ideal of $R$. Regard $\varphi$ as a map of $R^{m} \rightarrow R^{n}$, and put $M:=\operatorname{Coker} \varphi$. If $\operatorname{dim} R>n$, then by Satz 1 of [3], $n-t$ among the residues $\bar{e}_{1}, \ldots, \bar{e}_{n}$ of the canonical basis of $R^{n}$, say, $\bar{e}_{t+1}, \ldots, \bar{e}_{n}$, generate a free direct summand of rank $n-t$ in every localization $M_{\mathfrak{p}}, \mathfrak{p}$ nonmaximal. Therefore $M^{\prime}:=M / R \bar{e}_{t+1}+\cdots+R \bar{e}_{n}$ has finite length. Now $M^{\prime}$ is isomorphic to Coker $\varphi^{\prime}, \varphi^{\prime}$ consisting of the first $t$ columns of $\varphi$. $I_{t}\left(\varphi^{\prime}\right)$ is again primary to the maximal ideal of $R$, hence $\operatorname{dim} R<m-t+1$ by Theorem 2 (for the classical case of maximal minors). 
Faltings gives his theorem in a more general setting. For complete local domains the inequality of Theorem 3 becomes

$$
\text { ht } \mathrm{I}_{t}(\varphi)<\max (m+\operatorname{embdim} R-\operatorname{dim} R, n-t+1),
$$

embdim $R$ denoting the embedding dimension of $R$, i.e., the minimal number of generators of the maximal ideal of $R$. For the most general case cf. [3].

\section{REFERENCES}

1. J. A. Eagon and D. G. Northcott, Ideals defined by matrices and a certain complex associated with them, Proc. Roy. Soc. London Ser. A 269 (1962), 188-204.

2. D. Eisenbud and E. G. Evans, Jr., A generalized principal ideal theorem, Nagoya Math. J. 62 (1976), 41-53.

3. G. Faltings, Ein Kriterium für vollständige Durchschnitte, Invent. Math. 62 (1981), 383-402.

4. M. Hochster, Deep local rings, preprint, Aarhus, 1973.

5. __ Principal ideal theorems, Ring Theory (Waterloo, 1978), Lecture Notes in Math., vol. 734, Springer-Verlag, Berlin and New York, 1979.

6. I. Kaplansky, Commutative rings, rev. ed., The University of Chicago Press, Chicago and London, 1974.

7. H. Matsumura, Commutative algebra, Benjamin, New York, 1970.

8. G. Scheja and U. Storch, Differentielle Eigenschaften der Lokalisierungen analytischer Algebren, Math. Ann. 197 (1972), 137-170.

9. U. Vetter, Zu einem Satz von G. Trautmann über den Rang gewisser kohärenter analytischer Moduln, Arch. Math. (Basel) 24 (1973), 158-161.

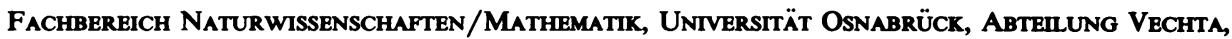
Vechta, Federal Republic of Germany 